\title{
The cortex is in overall control of 'voluntary' eye movement
}

\begin{abstract}
The neural circuits that control eye movements are complex and distributed in brainstem, basal ganglia, cerebellum, and multiple areas of cortex. The anatomical function of the substrates implicated in eye movements has been studied for decades in numerous countries, laboratories, and clinics. The modest goal of this brief review is twofold. (1) To present a focused overview of the knowledge about the role of the cerebral cortex in voluntary control of eye movements. (2) To very briefly mention two findings showing that the accepted hierarchy between the frontal and the occipital sensory areas involved in sensory-motor transformation might not be so trivial to reconcile, and to interpret in the context of eye movement command. This presentation has been part of the 44th Cambridge Ophthalmological Symposium, on ocular motility, 3 September 2014 to 5 November 2014.
\end{abstract}

Eye (2015) 29, 241-245; doi:10.1038/eye.2014.284; published online 5 December 2014

Historically, in humans, the data describing the implications of cortical areas in controlling eye movement have been collected in patients with lesions or degenerative diseases, whereas in animal models, two ancient but still preferred tools, for the delineation of cortical regions, involved the two techniques of low-threshold current stimulation and the recording of extracellular activity. ${ }^{1}$ More recently, ultrasonic ${ }^{2}$ and transcranial magnetic stimulations, ${ }^{3-5}$ as well as functional magnetic resonance imaging, provided complementary results to better understand with noninvasive techniques how eye movements are controlled by the brain. 6,7

Within the frontal cortex, the cortical structures of the saccadic systems include; the
P Pouget ${ }^{1,2,3}$

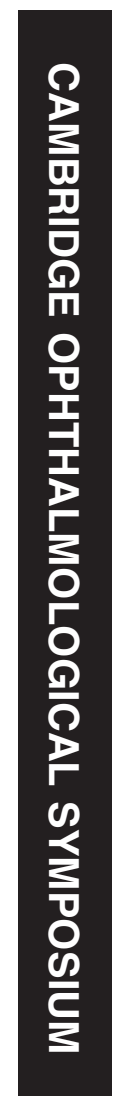

frontal eye field (FEF) and its role in saccade and pursuit eye movement control, the supplementary eye field (SEF) and the dorsolateral prefrontal cortex (dIPFC). The SEF and the dlPFC are both involved in the decisional processes that is governing ocular motor behavior, such as when and how they inhibit an unwanted reflexive saccade. The SEF and the dIPFC are also having a role in making eye movement at a memorized location, as well as producing sequences eye movements. In the temporoparietal regions, the activity of the posterior parietal cortex is less implicated in movement execution per se but more involved in visuospatial integration and attention. Lastly, the anterior cingulate cortex appears to be mainly and indirectly involved in the control of externally guided eye movements and attentional mechanisms.

As briefly introduced above, in the frontal lobe, three main areas are involved in eye movement control (Figure 1). The FEF is involved in the preparation and triggering of all saccades. ${ }^{8}$ In particular, FEF is involved in saccades generation, which are internally triggered toward a target already present (visually guided saccade), not yet present (predictive saccade), and no longer visible (memory-guided saccade) or located in the opposite direction (antisaccade). Despite some noticeable activity recorded in nonhuman primates, FEF region is believed to be less involved in the triggering of purely reflexive, visually guided saccades, which are externally triggered toward a stimulus appearing at a peripheral location. A subregion of FEF also controls pursuit eye movements, along with the cortex, the posterior temporoparietal areas, 9,10 and cerebellum. ${ }^{11}$ In humans, studies using fMRI have delimited the location of the FEF mainly to the intersection between the precentral sulcus and the superior frontal
${ }^{1}$ CNRS 7225, Paris, France

${ }^{2} \mathrm{ICM}$, Paris, France

${ }^{3}$ Université Pierre et Marie Curie, Paris, France

Correspondence: P Pouget, ICM, CNRS, INSERM, Université Pierre et Marie Curie, Hôpital de la Salpêtrière, 47 Boulevard de I'Hôpital, 75651 Paris CEDEX 13, France Tel/Fax: +33 (0) 1572743 57; E-mail: pierre.pouget@ upmc.fr

Received: 15 October 2014 Accepted in revised form: 23 October 2014 Published online: 5 December 2014 


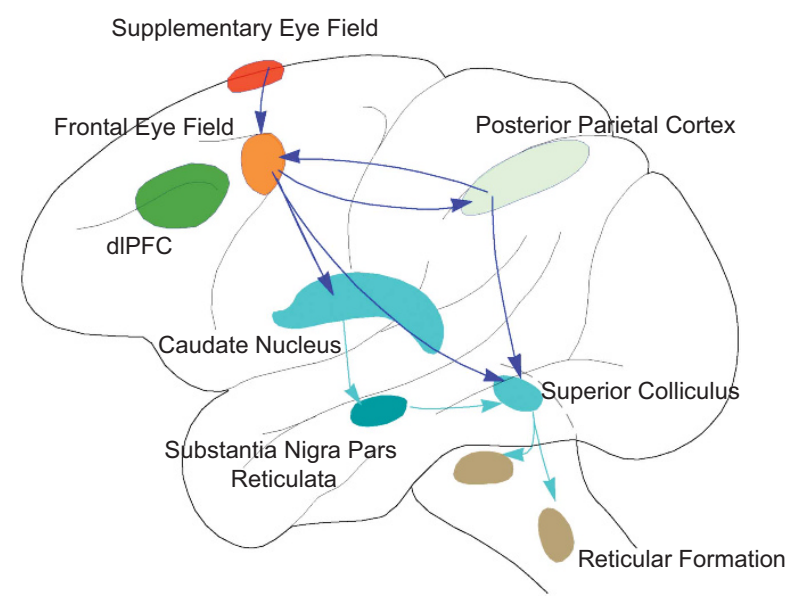

Figure 1 Supranuclear region implicated in the control of eye movements.

sulcus, whereas the pursuit-related area to a deeper region along the anterior wall, the fundus, and the deep part of the posterior wall. ${ }^{10}$ In the precentral sulcus, aside the main locus of the FEF, located at the junction with the superior frontal sulcus, another locus of activation is often observed in fMRI studies along the lateral part of this sulcus and the adjacent portion of the precentral gyrus. ${ }^{12}$ The specific role of this lateral locus remains to be determined, as it is activated both by single and combined eye and head movements. ${ }^{13}$

As illustrated in Figure 2, the antisaccade paradigm permits to study intentional saccades that have to be made in the direction opposite to a suddenly appearing peripheral visual target. ${ }^{14}$ To generate these movements at least two mechanisms are believed to be required: the inhibition of the unwanted reflexive saccade to the target; and the consecutive triggering of an intentional correct antisaccade made in the direction opposite to the target. Prosaccades and antisaccades have been intensively studied in humans (fMRI) and monkeys single unit (SU). In all reports, stronger activation has been observed just before antisaccades in the FEF than in prosaccade. ${ }^{15,16}$ All these studies, confirm that such intentional saccades require an early preparation of FEF in antisaccades but not in prosaccades. However and although FEF is active in antisaccade preparation, these results do not demonstrate that inhibition or relocation of misdirected reflexive prosaccades are organized within the FEF per se. In fact, activation of the dIPFC and/or the SEF were also recorded just before antisaccades. ${ }^{16}$ In that scenario, the inhibition of reflexive saccades by the dIPFC and/or SEF could be exerted via FEF or directly on the superior colliculus, without involving other cortical areas, via a prefrontocollicular tract. ${ }^{17}$

SEF is located on the medial surface of the superior frontal gyrus. ${ }^{16}$ In humans, SEF is located in the upper part of the para-central sulcus (Figure 1). Anatomically

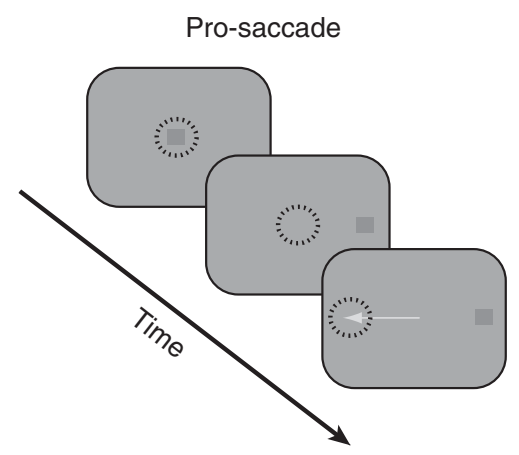

Memory-guided saccade
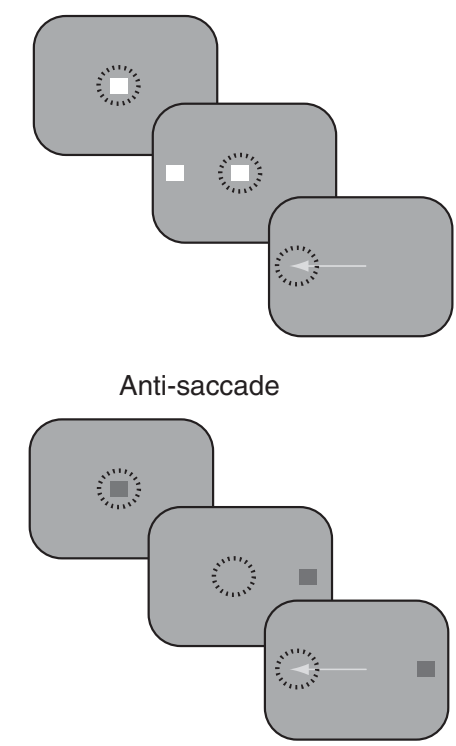

Figure 2 Saccade and saccadic paradigms.

SEF appears to be a critical node in the oculomotor circuit. SEF is connected with all areas involved in eye movement control-the FEF, the dlPFC, the anterior cingulate cortex (ACC), most of the parietal cortex, and also deep oculomotor structures in the brainstem. Electrical stimulation in monkey and lesion studies in humans have demonstrated that SEF is involved in motor program comprising a saccade combined with a body movement. ${ }^{18}$ In SU-recording studies in monkey, using a saccade sequence, it was demonstrated that the SEF neurons are also involved in the coding of temporally ordered saccadic eye movements. ${ }^{19}$ In the same vein, a recent TMS study in humans has shown that stimulation applied over the SEF resulted in a disruption of the saccade order in a double-step paradigm (comprising a sequence of two successive saccades). Altogether, these results validate that the SEF control and have an important role in motor preparation rather than motor program by itself, even when they are limited to a single saccade. ${ }^{8}$ 
Slightly more rostral and lateral than the SEF (dlPFC Figure 1), the dlPFC is involved in saccade inhibition, ${ }^{20}$ but see, ${ }^{21}$ as well as, short-term spatial memory and in decision processes. ${ }^{22}$ Human and nonhuman primates studies have demonstrated that the dIPFC, and, more particularly, area 46 of Brodmann and the adjacent Brodmann area 9, both located in the middle frontal gyrus, are involved in the control of memory-guided saccades. ${ }^{23}$ Aside its role in memory-guided saccades the dlPFC is also involved in the control of predictive saccades. ${ }^{24}$ These results in conjunction with those referred to above suggest that the dIPFC has a crucial role in decisional processes governing eye movement behavior, preparing intentional saccades by inhibiting unwanted reflexive saccades (inhibition), maintaining memorized information for forthcoming intentional saccades (short-term spatial memorization), or facilitating intentional anticipatory saccades (prediction), depending upon current external environmental and internal circumstances.

All these decisional rules that are important in guiding or inhibiting future responses are believed to be exerted through inhibitory interactions between neurons in the dIPFC, FEF, SEF, and parietal regions. In particular, the control of the timing during cognitive operations might be thereby shaped by the temporal flow of information between FEF, SEF, and dIPFC. ${ }^{25}$

In the parietal lobe, the location and function of subregions involved in eye movements and attention have been studied intensively, but are still not so well known. ${ }^{26,27}$ The parietal lobe and more particularly its posterior part, the PPC, are involved in the control of saccades and attention. In humans and nonhuman primates, the PPC includes the intraparietal sulcus (IPS) extending from the post-central sulcus anteriorly to the parieto-occipital sulcus posteriorly (Figure 1). The anatomy of the IPS is not trivial to compare across subjects and species, being relatively variable from one subject to another. ${ }^{28}$ The human parietal-eye-field (PEF) corresponds to the lateral intraparietal area of the monkey. The lateral intraparietal area is involved in the control of saccades, but also in attentional processes. Furthermore, low threshold of electrical stimulation of this area in the monkey or human results in a simple shift of visual attention (without eye movement), whereas stronger stimulation results in a saccade. ${ }^{29-31}$ These results emphasize in PPC but also in other brain regions, the close but distinct circuits existing between saccades and attention, even in the same area. ${ }^{32-34}$ The PEF appears to be located along the IPS, within the sulcus, in its posterior half, adjacent laterally to the anterior part of the angular gyrus (Brodmann area 39) and medially to the posterior part of the SPL (Brodmann area 7).

As it has been noticed in SEF, the activation of the PEF is also modulated by head position. The PEF projects to both the FEF and the superior colliculus (Figure 1). In the monkey, these two projections appear to be qualitatively different, with a more visual involvement for the parietoFEF projection and a more saccadic involvement for the parietosuperior colliculus projection. The parieto-FEF projection is believed to be mainly involved in visual processing, whereas the parietocolliculus projection is more involved in express saccades. ${ }^{32}$ The results of studies in patients with lesions affecting the posterior part of the internal capsule, damaging the direct parietocollicular tract originating in the PEF, are in accordance with the experimental results and confirm that the PEF is crucial for reflexive saccade generation, but not for intentional saccade generation. The latter depends mainly upon FEF, SEF, and dIPFC activities. ${ }^{25}$

Finally, the cingulate cortex, which is not per se an oculomotor area, has also been found to have an important role in the control of voluntary saccades. In particular, the rostral part of the cingulate cortex is involved in intentional saccade control, but not in reflexive saccade control. The dorsal bank of the ACC is involved, via direct connections to SEF, in preparing all the frontal ocular motor areas involved in intentional saccade control to act in the forthcoming motor behavior. The dIPFC is also connected to ACC. ${ }^{35,36}$

\section{Discussion}

In the illustrated classical view of this brief document, it is argued that the signal sufficient to elicit reflexive saccades are largely generated by the short loop, through the posterior part of the internal capsule, between the parietal and the superior colliculus, whereas voluntary eye movements required the additive and active signal from frontomedian regions including FEF, dlPFC, SEF, and ACC. However, some findings suggest that saccades might be generated in a much more intermixed activities than previously thought.

In 1991, Felleman and Van Essen ${ }^{37}$ have proposed a hierarchical model of visual processing that is largely accepted. In this model, visual information reaches the frontal cortex and FEF in particular, at a very high level (which could be functionally transcript by a delayed visual response in these frontal areas). Although, these conceptions did account for psychophysiological models and hypotheses, important functional contradictions have been reported but rarely highlighted in particular in the oculomotor literature. First, recent experiments have demonstrated that the neuronal activities in monkeys, but also in humans, can reach the frontal part of the cortex and FEF in particular only after $60 \mathrm{~ms}$ (latencies comparable to early response in visual cortex). ${ }^{38-40}$ Second and rarely accounted for in the literature of express saccades, some anatomical findings provide 
evidence that part of the activity in the frontal cortex mainly drive, via connections to extrastriate visual areas, feedforward signals and not, as often reported, feedback signals. $^{32,41}$

\section{Conclusion}

All the accumulated studies in humans and nonhuman primates have been extremely important to describe the cortical control of eye movements. At the present day, in the frontal cortex, FEF is believed to be involved for the execution of intentional saccades. The neuronal responses present in FEF are prepared by the activities in SEF, ACC, and dIPFC, whereas in the parietal lobe, short loops, through the posterior part of the internal capsule, between the parietal and the superior colliculus, are believed to be sufficient to elicit reflexive saccades. However, to mention only two findings that are going against the grain in the literature of eye movements control: (1) the hierarchy between the frontal, the parietal, and the sensory areas might have been too simplified; (2) the antomicofunctional constrains present for the sensory-motor transformation might not be so trivial to reconcile with the current hierarchy of visual areas. Despite tremendous efforts and many successful findings, future researches are still required to causally disentangle the roles and hierarchies of the cortical and subcortical areas responsible for the control of voluntary eye movements.

\section{Conflict of interest}

The author declares no conflict of interest.

\section{References}

1 Lemon R. Methods for neuronal recording in conscious animals. John Wiley and Sons: Chichester, UK; 1984

2 Deffieux T, Younan Y, Wattiez N, Tanter M, Pouget P, Aubry JF. Low-intensity focused ultrasound modulates monkey visuomotor behavior. Curr Biol 2013; 23(23): 2430-2433.

3 Gerits A, Ruff CC, Guipponi O, Wenderoth N, Driver J, Vanduffel W. Transcranial magnetic stimulation of macaque frontal eye fields decreases saccadic reaction time. Exp Brain Res 2011; 212(1): 143-152.

4 Müri RM, Nyffeler T. Neurophysiology and neuroanatomy of reflexive and volitional saccades as revealed by lesion studies with neurological patients and transcranial magnetic stimulation (TMS). Brain Cogn 2008; 68(3): 284-292.

5 Valero-Cabre A, Wattiez N, Monfort M, François C, RivaudPéchoux S, Gaymard B et al. Frontal non-invasive neurostimulation modulates antisaccade preparation in non-human primates. PLoS One 2012; 7(6): e38674.

6 Roberts DC, Marcelli V, Gillen JS, Carey JP, Della Santina CC, Zee DS. MRI magnetic field stimulates rotational sensors of the brain. Curr Biol 2011; 21(19): 1635-1640.
7 Ohayon S, Grimaldi P, Schweers N, Tsao DY. Saccade modulation by optical and electrical stimulation in the macaque frontal eye field. J Neurosci 2013; 33(42): 16684-16697.

8 Schall JD. Neural basis of saccade target selection. Rev Neurosci 1995; 6(1): 63-85.

9 Izawa Y, Suzuki H. Activity of fixation neurons in the monkey frontal eye field during smooth pursuit eye movements. J Neurophysiol 2014; 112(2): 249-262.

$10 \mathrm{Li} \mathrm{JX}$, Lisberger SG. Learned timing of motor behavior in the smooth eye movement region of the frontal eye fields. Neuron 2011; 69(1): 159-169.

11 Leigh RJ, Zee DS. The Neurology of Eye Movements, 4th ed. Oxford University Press: New York; 2006.

12 Lachaux JP, Hoffmann D, Minotti L, Berthoz A, Kahane P. Intracerebral dynamics of saccade generation in the human frontal eye field and supplementary eye field. Neuroimage 2006; 30(4): 1302-1312.

13 Thura D, Hadj-Bouziane F, Meunier M, Boussaoud D. Hand modulation of visual, preparatory, and saccadic activity in the monkey frontal eye field. Cereb Cortex 2011; 21(4): 853-864.

14 Antoniades C, Ettinger U, Gaymard B, Gilchrist I, Kristjánsson A, Kennard C et al. An internationally standardised antisaccade protocol. Vision Res 2013; 84: 1-5.

15 Brown MR, Vilis T, Everling S. Frontoparietal activation with preparation for antisaccades. J Neurophysiol 2007; 98(3): 1751-1762.

16 Schlag-Rey M, Amador N, Sanchez H, Schlag J. Antisaccade performance predicted by neuronal activity in the supplementary eye field. Nature 1997; 390(6658): 398-401.

17 Johnston K, Everling S. Monkey dorsolateral prefrontal cortex sends task-selective signals directly to the superior colliculus. J Neurosci 2006; 26(48): 12471-12478.

18 Chapman BB, Pace MA, Cushing SL, Corneil BD. Recruitment of a contralateral head turning synergy by stimulation of monkey supplementary eye fields. J Neurophysiol 2012; 107(6): 1694-1710.

19 Chen LL, Wise SP. Neuronal activity in the supplementary eye field during acquisition of conditional oculomotor associations. J Neurophysiol 1995; 73(3): 1101-1121.

20 Pierrot-Deseilligny CH, Müri RM, Nyffeler T, Milea D. The role of the human dorsolateral prefrontal cortex in ocular motor behavior. Ann NY Acad Sci 2005; 1039: 239-251.

21 Johnston K, Koval MJ, Lomber SG, Everling S. Macaque dorsolateral prefrontal cortex does not suppress saccaderelated activity in the superior colliculus. Cereb Cortex 2014; 24(5): 1373-1388.

22 Levy R, Goldman-Rakic PS. Segregation of working memory functions within the dorsolateral prefrontal cortex. Exp Brain Res 2000; 133(1): 23-32.

23 Selemon LD, Goldman-Rakic PS. Common cortical and subcortical targets of the dorsolateral prefrontal and posterior parietal cortices in the rhesus monkey: evidence for a distributed neural network subserving spatially guided behavior. J Neurosci 1988; 8(11): 4049-4068.

24 Pierrot-Deseilligny C, Müri RM, Ploner CJ, Gaymard B, Demeret S, Rivaud-Pechoux S. Decisional role of the dorsolateral prefrontal cortex in ocular motor behaviour. Brain 2003; 126(Pt 6): 1460-1473.

25 Schall JD. Production, control, and visual guidance of saccadic eye movements. ISRN Neurol 2013; 2013: 752384. 
26 Ibos G, Duhamel JR, Ben Hamed S. A functional hierarchy within the parietofrontal network in stimulus selection and attention control. J Neurosci 2013; 33(19): 8359-8369.

27 Bisley JW, Goldberg ME. Attention, intention, and priority in the parietal lobe. Annu Rev Neurosci 2010; 33: 1-21.

28 Caspers S, Geyer S, Schleicher A, Mohlberg H, Amunts K, Zilles $\mathrm{K}$. The human inferior parietal cortex: cytoarchitectonic parcellation and interindividual variability. Neuroimage 2006; 33(2): 430-448.

29 Foley NC, Jangraw DC, Peck C, Gottlieb J. Novelty enhances visual salience independently of reward in the parietal lobe. J Neurosci 2014; 34(23): 7947-7957.

30 Mirpour K, Ong WS, Bisley JW. Microstimulation of posterior parietal cortex biases the selection of eye movement goals during search. J Neurophysiol 2010; 104(6): 3021-3028.

31 Desmurget M, Reilly KT, Richard N, Szathmari A, Mottolese C, Sirigu A. Movement intention after parietal cortex stimulation in humans. Science 2009; 324(5928): 811-813.

32 Pouget P, Stepniewska I, Crowder EA, Leslie MW, Emeric $\mathrm{EE}$, Nelson MJ et al. Visual and motor connectivity and the distribution of calcium-binding proteins in macaque frontal eye field: implications for saccade target selection. Front Neuroanat 2009; 3: 2.

33 Doré-Mazars K, Pouget P, Beauvillain C. Attentional selection during preparation of eye movements. Psychol Res 2004; 69(1-2): 67-76.

34 Kincade JM, Abrams RA, Astafiev SV, Shulman GL, Corbetta M. An event-related functional magnetic resonance imaging study of voluntary and stimulus-driven orienting of attention. J Neurosci 2005; 25(18): 4593-4604.

35 Darian-Smith C, Tan A, Edwards S. Comparing thalamocortical and corticothalamic microstructure and spatial reciprocity in the macaque ventral posterolateral nucleus (VPLc) and medial pulvinar. J Comp Neurol 1999; 410: 211-234.

36 Koval MJ, Hutchison RM, Lomber SG, Everling S. Effects of unilateral deactivations of dorsolateral prefrontal cortex and anterior cingulate cortex on saccadic eye movements. J Neurophysiol 2014; 111(4): 787-803.

37 Felleman DJ, Van Essen DC. Distributed hierarchical processing in the primate cerebral cortex. Cereb Cortex 1991; 1(1): 1-47.

38 Pouget P, Emeric EE, Stuphorn V, Reis K, Schall JD. Chronometry of visual responses in frontal eye field, supplementary eye field, and anterior cingulate cortex. J Neurophysiol 2005; 94(3): 2086-2092.

39 Kirchner H, Barbeau EJ, Thorpe SJ, Régis J, LiégeoisChauvel C. Ultra-rapid sensory responses in the human frontal eye field region. J Neurosci 2009; 29(23): 7599-7606.

40 Schmolesky MT, Wang Y, Hanes DP, Thompson KG, Leutgeb S, Schall JD et al. Signal timing across the macaque visual system. J Neurophysiol 1998; 79(6): 3272-3278.

41 Barone P, Batardiere A, Knoblauch K, Kennedy H. Laminar distribution of neurons in extrastriate areas projecting to visual areas V1 and V4 correlates with the hierarchical rank and indicates the operation of a distance rule. J Neurosci 2000; 20(9): 3263-3281. 